\title{
TWO NON-CLOSURE PROPERTIES ON THE CLASS OF SUBEXPONENTIAL DENSITIES
}

\author{
TOSHIRO WATANABE AND KOUJI YAMAMURO
}

\begin{abstract}
Relations between subexponential densities and locally subexponential distributions are discussed. It is shown that the class of subexponential densities is neither closed under convolution roots nor closed under asymptotic equivalence. A remark is given on the closure under convolution roots for the class of convolution equivalent distributions.
\end{abstract}

Key words or phrases: subexponential densities, local subexponentiality, convolution roots, asymptotic equivalence

Mathematics Subject Classification: 60E99, 60G50

\section{INTRODUCTION AND MAIN RESULTS}

In what follows, we denote by $\mathbb{R}$ the real line and by $\mathbb{R}_{+}$the half line $[0, \infty)$. Let $\mathbb{N}$ be the totality of positive integers. The symbol $\delta_{a}(d x)$ stands for the delta measure at $a \in \mathbb{R}$. Let $\eta$ and $\rho$ be probability measures on $\mathbb{R}$. We denote the convolution of $\eta$ and $\rho$ by $\eta * \rho$ and denote $n$-th convolution power of $\rho$ by $\rho^{n *}$. Let $f(x)$ and $g(x)$ be integrable functions on $\mathbb{R}$. We denote by $f^{n \otimes}(x) n$-th convolution power of $f(x)$ and by $f \otimes g(x)$ the convolution of $f(x)$ and $g(x)$. For positive functions $f_{1}(x)$ and $g_{1}(x)$ on $[a, \infty)$ for some $a \in \mathbb{R}$, we define the relation $f_{1}(x) \sim g_{1}(x)$ by $\lim _{x \rightarrow \infty} f_{1}(x) / g_{1}(x)=1$. We also define the relation $a_{n} \sim b_{n}$ for positive sequences $\left\{a_{n}\right\}_{n=A}^{\infty}$ and $\left\{b_{n}\right\}_{n=A}^{\infty}$ with $A \in \mathbb{N}$ by $\lim _{n \rightarrow \infty} a_{n} / b_{n}=1$. We define the class $\mathcal{P}_{+}$as the totality of probability distributions on $\mathbb{R}_{+}$. In this paper, we prove that the class of subexponential densities is not closed under two important closure properties. We say that a measurable function $g(x)$ on $\mathbb{R}$ is a density function if $\int_{-\infty}^{\infty} g(x) d x=1$ and $g(x) \geq 0$ for all $x \in \mathbb{R}$.

Definition 1.1. (i) A nonnegative measurable function $g(x)$ on $\mathbb{R}$ belongs to the class $\mathbf{L}$ if $g(x)>0$ for all sufficiently large $x>0$ and if $g(x+a) \sim g(x)$ for any $a \in \mathbb{R}$.

T. Watanabe: Center for Mathematical Sciences The Univ. of Aizu. Aizu-Wakamatsu 965-8580, Japan. e-mail: t-watanb@u-aizu.ac.jp

K. Yamamuro: Faculty of Engineering Gifu Univ. Gifu 501-1193, Japan.

e-mail: yamamuro@gifu-u.ac.jp 
(ii) A measurable function $g(x)$ on $\mathbb{R}$ belongs to the class $\mathcal{L}_{d}$ if $g(x)$ is a density function and $g(x) \in \mathbf{L}$.

(iii) A measurable function $g(x)$ on $\mathbb{R}$ belongs to the class $\mathcal{S}_{d}$ if $g(x) \in \mathcal{L}_{d}$ and $g \otimes g(x) \sim 2 g(x)$

(iv) A distribution $\rho$ on $\mathbb{R}$ belongs to the class $\mathcal{L}_{a c}$ if there is $g(x) \in \mathcal{L}_{d}$ such that $\rho(d x)=g(x) d x$.

(v) A distribution $\rho$ on $\mathbb{R}$ belongs to the class $\mathcal{S}_{a c}$ if there is $g(x) \in \mathcal{S}_{d}$ such that $\rho(d x)=g(x) d x$.

Densities in the class $\mathcal{S}_{d}$ are called subexponential densities and those in the class $\mathcal{L}_{d}$ are called long-tailed densities. The study on the class $\mathcal{S}_{d}$ goes back to Chover et al. [2]. Let $\rho$ be a distribution on $\mathbb{R}$. Note that $c^{-1} \rho((x-c, x])$ is a density function on $\mathbb{R}$ for every $c>0$.

Definition 1.2. (i) Let $\Delta:=(0, c]$ with $c>0$. A distribution $\rho$ on $\mathbb{R}$ belongs to the class $\mathcal{L}_{\Delta}$ if $\rho((x, x+c]) \in \mathbf{L}$.

(ii) Let $\Delta:=(0, c]$ with $c>0$. A distribution $\rho$ on $\mathbb{R}$ belongs to the class $\mathcal{S}_{\Delta}$ if $\rho \in \mathcal{L}_{\Delta}$ and $\rho * \rho((x, x+c]) \sim 2 \rho((x, x+c])$.

(iii) A distribution $\rho$ on $\mathbb{R}$ belongs to the class $\mathcal{L}_{\text {loc }}$ if $\rho \in \mathcal{L}_{\Delta}$ for each $\Delta:=(0, c]$ with $c>0$.

(iv) A distribution $\rho$ on $\mathbb{R}$ belongs to the class $\mathcal{S}_{l o c}$ if $\rho \in \mathcal{S}_{\Delta}$ for each $\Delta:=(0, c]$ with $c>0$.

(v) A distribution $\rho \in \mathcal{L}_{\text {loc }}$ belongs to the class $\mathcal{U} \mathcal{L}_{\text {loc }}$ if there exists $p(x) \in \mathcal{L}_{d}$ such that $c^{-1} \rho((x-c, x]) \sim p(x)$ uniformly in $c \in(0,1]$.

(vi) A distribution $\rho \in \mathcal{S}_{\text {loc }}$ belongs to the class $\mathcal{U S}_{\text {loc }}$ if there exists $p(x) \in \mathcal{S}_{d}$ such that $c^{-1} \rho((x-c, x]) \sim p(x)$ uniformly in $c \in(0,1]$.

Distributions in the class $\mathcal{S}_{\text {loc }}$ are called locally subexponential, those in the class $\mathcal{U} S_{l o c}$ are called uniformly locally subexponential. The class $\mathcal{S}_{\Delta}$ was introduced by Asmussen et al. [1] and the class $\mathcal{S}_{l o c}$ was by Watanabe and Yamamuro [14. Detailed acounts of the classes $\mathcal{S}_{d}$ and $\mathcal{S}_{\Delta}$ are found in the book of Foss et al. [6]. First, we present some interesting results on the classes $\mathcal{S}_{d}$ and $\mathcal{S}_{l o c}$.

Proposition 1.1. We have the following.

(i) Let $\Delta:=(0, c]$ with $c>0$ and let $p(x):=c^{-1} \mu((x-c, x])$ for a distribution $\mu$ on $\mathbb{R}_{+}$. Then $\mu \in \mathcal{S}_{\Delta}$ if and only if $p(x) \in \mathcal{S}_{d}$. Moreover, $\mu \in \mathcal{S}_{\text {loc }} \cap \mathcal{P}_{+}$if and only if there exists a density function $q(x)$ on $\mathbb{R}_{+}$such that $q(x) \in \mathcal{S}_{d}$ and $c^{-1} \mu((x-c, x]) \sim q(x)$ for every $c>0$.

(ii) Let $\rho_{1}(d x):=q_{1}(x) d x$ be a distribution on $\mathbb{R}_{+}$. If $q_{1}(x)$ is continuous with compact support and if $\rho_{2} \in \mathcal{S}_{l o c} \cap \mathcal{P}_{+}$, then $\rho_{1} * \rho_{2}(d x)=\left(\int_{0-}^{x+} q_{1}(x-u) \rho_{2}(d u)\right) d x$ and $\int_{0-}^{x+} q_{1}(x-u) \rho_{2}(d u) \in \mathcal{S}_{d}$.

(iii) Let $\mu$ be a distribution on $\mathbb{R}_{+}$. If there exist distributions $\rho_{c}$ for $c>0$ such that, for every $c>0$, the support of $\rho_{c}$ is included in $[0, c]$ and $\rho_{c} * \mu \in \mathcal{S}_{\text {loc }}$, then $\mu \in \mathcal{S}_{l o c}$. 
Definition 1.3. (i) We say that a class $\mathcal{C}$ of probability distributions on $\mathbb{R}$ is closed under convolution roots if $\mu^{n *} \in \mathcal{C}$ for some $n \in \mathbb{N}$ implies that $\mu \in \mathcal{C}$.

(ii) Let $p_{1}(x)$ and $p_{2}(x)$ be density functions on $\mathbb{R}$. We say that a class $\mathcal{C}$ of density functions is closed under asymptotic equivalence if $p_{1}(x) \in \mathcal{C}$ and $p_{2}(x) \sim c p_{1}(x)$ with $c>0$ implies that $p_{2}(x) \in \mathcal{C}$.

The class $\mathcal{S}_{a c}$ is a proper subclass of the class $\mathcal{U S}_{\text {loc }}$ because a distribution in $\mathcal{U S}_{\text {loc }}$ can have a point mass. Moreover, the class $\mathcal{U S}_{\text {loc }}$ is a proper subclass of the class $\mathcal{S}_{l o c}$ as the following theorem shows.

Theorem 1.1. There exists a distribution $\mu \in \mathcal{S}_{l o c} \backslash \mathcal{U} \mathcal{S}_{l o c}$ such that $\mu^{2 *} \in \mathcal{S}_{a c}$.

Corollary 1.1. We have the following.

(i) The class $\mathcal{S}_{a c}$ is not closed under convolution roots.

(ii) The class $\mathcal{U S}_{\text {loc }}$ is not closed under convolution roots.

(iii) The class $\mathcal{L}_{a c}$ is not closed under convolution roots.

(iv) The class $\mathcal{U L}_{\text {loc }}$ is not closed under convolution roots.

The class $\mathcal{S}_{d}$ is closed under asymptotic equivalence in the one-sided case. See (ii) of Lemma 2.1 below. However, Foss et al. [6] suggest the possibility of non-closure under asymptotic equivalence for the class $\mathcal{S}_{d}$ in the two-sided case. We exactly prove it as follows.

Theorem 1.2. The class $\mathcal{S}_{d}$ is not closed under asymptotic equivalence, that is, there exist $p_{1}(x) \in \mathcal{S}_{d}$ and $p_{2}(x) \notin \mathcal{S}_{d}$ such that $p_{2}(x) \sim c p_{1}(x)$ with $c>0$.

In Sect. 2, we prove Proposition 1.1. In Sect. 3, we prove Theorems 1.1 and 1.2. In Sect. 4, we give a remark on the closure under convolution roots.

\section{Proof of Proposition 1.1}

We present two lemmas for the proofs of main results and then prove Proposition 1.1.

Lemma 2.1. Let $f(x)$ and $g(x)$ be density functions on $\mathbb{R}_{+}$.

(i) If $f(x) \in \mathcal{L}_{d}$, then $f^{n \otimes}(x) \in \mathcal{L}_{d}$ for every $n \in \mathbb{N}$.

(ii) If $f(x) \in \mathcal{S}_{d}$ and $g(x) \sim c f(x)$ with $c>0$, then $g(x) \in \mathcal{S}_{d}$.

(iii) Assume that $f(x) \in \mathcal{L}_{d}$. Then, $f(x) \in \mathcal{S}_{d}$ if and only if

$$
\lim _{A \rightarrow \infty} \limsup _{x \rightarrow \infty} \frac{1}{f(x)} \int_{A}^{x-A} f(x-u) f(u) d u=0 .
$$

Proof Proof of assertion (i) is due to Theorem 4.3 of [6]. Proofs of assertions (ii) and (iii) are due to Theorems 4.8 and 4.7 of [6], respectively.

Lemma 2.2. (i) Let $\Delta:=(0, c]$ with $c>0$. Assume that $\rho \in \mathcal{L}_{\Delta} \cap \mathcal{P}_{+}$. Then, $\rho \in \mathcal{S}_{\Delta}$ if and only if

$$
\lim _{A \rightarrow \infty} \limsup _{x \rightarrow \infty} \frac{1}{\rho((x, x+c])} \int_{A+}^{(x-A)-} \rho((x-u, x+c-u]) \rho(d u)=0 .
$$


(ii) Assume that $\rho \in \mathcal{L}_{\text {loc }} \cap \mathcal{P}_{+}$. Then, $\rho^{n *} \in \mathcal{L}_{\text {loc }}$ for every $n \in \mathbb{N}$. Moreover, $\rho((x-c, x]) \sim c \rho((x-1, x])$ for every $c>0$.

(iii) Let $\rho_{2} \in \mathcal{P}_{+}$. If $\rho_{1} \in \mathcal{S}_{l o c} \cap \mathcal{P}_{+}$and $\rho_{2}((x-c, x]) \sim c_{1} \rho_{1}((x-c, x])$ with $c_{1}>0$ for every $c>0$, then $\rho_{2} \in \mathcal{S}_{\text {loc }} \cap \mathcal{P}_{+}$.

Proof Proof of assertion (i) is due to Theorem 4.21 of [6]. First assertion of (ii) is due to Corollary 4.19 of [6]. Second one is proved as (2.6) in Theorem 2.1 of [14]. Proof of assertion (iii) is due to Theorem 4.22 of [6].

Proof of (i) of Proposition $1.1 \quad$ Let $\rho(d x):=c^{-1} 1_{[0, c)}(x) d x$. First, we prove that if $\mu \in \mathcal{S}_{\text {loc }} \cap \mathcal{P}_{+}$, then $\rho * \mu \in \mathcal{S}_{a c}$. We can assume that $c=1$. Suppose that $\mu \in \mathcal{S}_{l o c}$. Let $p(x):=\mu((x-1, x])$. We have $\rho * \mu(d x)=\mu((x-1, x]) d x$ and hence $p(x) \in \mathcal{L}_{d}$. Let $A$ be a positive integer and let $X, Y$ be independent random variables with the same distribution $\mu$. Then, we have for $x>2 A+2$

$$
\begin{aligned}
& \int_{A}^{x-A} p(x-u) p(u) d u \\
& =2 \int_{A}^{x / 2} p(x-u) p(u) d u \\
& =2 \int_{A}^{x / 2} P(x-u-1<X \leq x-u, u-1<Y \leq u) d u \\
& \leq 2 \int_{A}^{x / 2} P(X>A, Y>A, x-2<X+Y \leq x, u-1<Y \leq u) d u \\
& \leq 2 \sum_{n=A}^{\infty} \int_{n}^{n+1} P(X>A, Y>A, x-2<X+Y \leq x, n-1<Y \leq n+1) d u \\
& \leq 4 P(X>A, Y>A, x-2<X+Y \leq x) \\
& \leq 4 \int_{A+}^{(x-A)-} \mu((x-2-u, x-u]) \mu(d u) .
\end{aligned}
$$

Since $\mu \in \mathcal{S}_{l o c}$, we obtain from (i) of Lemma 2.2 that

$$
\lim _{A \rightarrow \infty} \limsup _{x \rightarrow \infty} \frac{\int_{A}^{x-A} p(x-u) p(u) d u}{p(x)}=0 .
$$

Thus, we see from (iii) of Lemma 2.1 that $p(x) \in \mathcal{S}_{d}$.

Conversely, suppose that $p(x) \in \mathcal{S}_{d}$. Then, we have $\mu \in \mathcal{L}_{\Delta}$. Let $[y]$ be the largest integer not exceeding a real number $y$. Choose sufficiently large integer $A>0$. Note that there are positive constants $c_{j}$ for $1 \leq j \leq 4$ such that

$$
c_{1} p(x-n) \leq p(x-u) \leq c_{2} p(x-n) \text { and } c_{3} p(n) \leq p(u) \leq c_{4} p(n)
$$


for $n \leq u \leq n+1, A \leq n \leq[x+1-A]$, and $x>2 A+2$. Thus, we find that

$$
\begin{aligned}
& P(A<X, A<Y, x<X+Y \leq x+1) \\
& \leq \sum_{n=A}^{[x+1-A]} \int_{n}^{n+1} \mu((x-u, x+1-u]) \mu(d u) \\
& =\sum_{n=A}^{[x+1-A]} \int_{n}^{n+1} p(x-u+1) \mu(d u) \\
& \leq c_{2} \sum_{n=A}^{[x+1-A]} p(x-n+1) p(n+1) \\
& \leq \frac{c_{2}}{c_{1} c_{3}} \sum_{n=A}^{[x+1-A]} \int_{n}^{n+1} p(x-u+1) p(u+1) d u \\
& \leq \frac{c_{2}}{c_{1} c_{3}} \int_{A}^{x+2-A} p(x-u+1) p(u+1) d u
\end{aligned}
$$

Since $p(x) \in \mathcal{S}_{d}$, we establish from (iii) of Lemma 2.1 that

$$
\limsup _{A \rightarrow \infty} \limsup _{x \rightarrow \infty} \frac{P(A<X, A<Y, x<X+Y \leq x+1)}{P(x<X \leq x+1)}=0 .
$$

Thus, $\mu \in \mathcal{S}_{\Delta}$ by (i) of Lemma 2.2. Note from (ii) of Lemma 2.2 that if $\mu \in \mathcal{S}_{\text {loc }}$, then $c^{-1} \mu((x-c, x]) \sim \mu((x-1, x])$ for every $c>0$. Thus, the second assertion is true.

Proof of (ii) of Proposition 1.1 Suppose that $\rho_{1}(d x):=q_{1}(x) d x$ be a distribution on $\mathbb{R}_{+}$such that $q_{1}(x)$ is continuous with compact support in $[0, N]$. Let $q(x):=$ $\int_{0-}^{x+} q_{1}(x-u) \rho_{2}(d u)$. For $M \in \mathbb{N}$, there are $\delta(M)>0$ and $a_{n}=a_{n}(M) \geq 0$ for $n \in \mathbb{N}$ such that $\lim _{M \rightarrow \infty} \delta(M)=0$ and $a_{n} \leq q_{1}(x) \leq a_{n}+\delta(M)$ for $M^{-1}(n-1)<x \leq M^{-1} n$ and $1 \leq n \leq M N$. Define $J(M ; x)$ as

$$
J(M ; x):=\sum_{n=1}^{M N} a_{n} \rho_{2}\left(\left(x-M^{-1} n, x-M^{-1}(n-1)\right]\right) .
$$

Then, we have

$$
J(M ; x) \sim \rho_{2}((x-1, x]) \sum_{n=1}^{M N} a_{n} M^{-1}
$$

and for $x>N$

$$
J(M ; x) \leq q(x) \leq J(M ; x)+\delta(M) \rho_{2}((x-N, x]) .
$$


Since $\lim _{M \rightarrow \infty} \delta(M)=0$ and

$$
\lim _{M \rightarrow \infty} \sum_{n=1}^{M N} a_{n} M^{-1}=\int_{0}^{N} q_{1}(x) d x=1,
$$

we obtain from (2.1) that

$$
q(x) \sim \rho_{2}((x-1, x]) .
$$

Since $\rho_{2} \in \mathcal{S}_{\text {loc }}$, we conclude from (i) of Proposition 1.1 that $q(x) \in \mathcal{S}_{d}$.

Proof of (iii) of Proposition 1.1 Suppose that the support of $\rho_{c}$ is included in $[0, c]$ and $\rho_{c} * \mu \in \mathcal{S}_{l o c}$ for every $c>0$. Let $X$ and $Y$ be independent random variables with the same distribution $\mu$, and let $X_{c}$ and $Y_{c}$ be independent random variables with the same distribution $\rho_{c}$. Define $J_{1}\left(c ; c_{1} ; a ; x\right)$ and $J_{2}\left(c ; c_{1} ; a ; x\right)$ for $a \in \mathbb{R}$ and $c_{1}>0$ as

$$
\begin{gathered}
J_{1}\left(c ; c_{1} ; a ; x\right):=\frac{P\left(x+a<X+X_{c} \leq x+c_{1}+a\right)}{P\left(x<X+X_{c} \leq x+c_{1}+c\right)}, \\
J_{2}\left(c ; c_{1} ; a ; x\right):=\frac{P\left(x+a<X+X_{c} \leq x+c_{1}+c+a\right)}{P\left(x<X+X_{c} \leq x+c_{1}\right)} .
\end{gathered}
$$

We see that

$$
J_{1}\left(c ; c_{1} ; a ; x\right) \leq \frac{P\left(x+a<X \leq x+c_{1}+a\right)}{P\left(x<X \leq x+c_{1}\right)} \leq J_{2}\left(c ; c_{1} ; a ; x\right) .
$$

Since $\rho_{c} * \mu \in \mathcal{L}_{l o c}$, we obtain that

$$
\lim _{x \rightarrow \infty} J_{1}\left(c ; c_{1} ; a ; x\right)=\frac{c_{1}}{c_{1}+c}
$$

and

$$
\lim _{x \rightarrow \infty} J_{2}\left(c ; c_{1} ; a ; x\right)=\frac{c_{1}+c}{c_{1}} .
$$

Thus, as $c \rightarrow 0$ we have by $(2.2)$

$$
\lim _{x \rightarrow \infty} \frac{P\left(x+a<X \leq x+c_{1}+a\right)}{P\left(x<X \leq x+c_{1}\right)}=1,
$$

and hence $\mu \in \mathcal{L}_{\text {loc }}$. We find from $\rho_{c} * \mu \in \mathcal{S}_{\text {loc }}$ and (i) of Lemma 2.2 that

$$
\begin{aligned}
& \lim _{A \rightarrow \infty} \limsup _{x \rightarrow \infty} \frac{P\left(X>A, Y>A, x<X+Y \leq x+c_{1}\right)}{P\left(x<X \leq x+c_{1}\right)} \\
& \leq \lim _{A \rightarrow \infty} \limsup _{x \rightarrow \infty} \frac{P\left(X>A, Y>A, x<X+X_{c}+Y+Y_{c} \leq x+c_{1}+2 c\right)}{P\left(x<X+X_{c} \leq x+c_{1}\right)}=0 .
\end{aligned}
$$

Thus, we see from (i) of Lemma 2.2 that $\mu \in \mathcal{S}_{\text {loc }}$. 


\section{Proofs of Theorems 1.1 And 1.2}

For the proofs of the theorems, we introduce a distribution $\mu$ as follows. Let $1<x_{0}<b$ and choose $\delta \in(0,1)$ satisfying $\delta<\left(x_{0}-1\right) \wedge\left(b-x_{0}\right)$. We take a continuous periodic function $h(x)$ on $\mathbb{R}$ with period $\log b$ such that $h(\log x)>0$ for $x \in\left[1, x_{0}\right) \cup\left(x_{0}, b\right]$ and

$$
h(\log x)= \begin{cases}0 & \text { for } x=x_{0}, \\ \frac{-1}{\log \left|x-x_{0}\right|} & \text { for each } x \text { with } 0<\left|x-x_{0}\right|<\delta .\end{cases}
$$

Let

$$
\phi(x):=x^{-\alpha-1} h(\log x) 1_{[1, \infty)}(x)
$$

with $\alpha>0$. Here, the symbol $1_{[1, \infty)}(x)$ stands for the indicator function of the set $[1, \infty)$. Define a distribution $\mu$ as

$$
\mu(d x):=M^{-1} \phi(x) d x,
$$

where $M:=\int_{1}^{\infty} x^{-1-\alpha} h(\log x) d x$.

Lemma 3.1. We have $\mu \in \mathcal{L}_{\text {loc }}$.

Proof Let $\left\{y_{n}\right\}$ be a sequence such that $1 \leq y_{n} \leq b$ and $\lim _{n \rightarrow \infty} y_{n}=y$ for some $y \in[1, b]$. Then, we put $x_{n}=b^{m_{n}} y_{n}$, where $m_{n}$ is a positive integer and $\lim _{n \rightarrow \infty} x_{n}=\infty$. In what follows, $c>0$ and $c_{1} \geq 0$.

Case 1. Suppose that $y \neq x_{0}$. Let $x_{n}+c_{1} \leq u \leq x_{n}+c_{1}+c$. Then, we have

$$
y_{n}+b^{-m_{n}} c_{1} \leq b^{-m_{n}} u \leq y_{n}+b^{-m_{n}}\left(c_{1}+c\right) \text {, }
$$

and thereby $\lim _{n \rightarrow \infty} b^{-m_{n}} u=y$. This yields that

$$
h(\log u)=h\left(\log \left(b^{-m_{n}} u\right)\right) \sim h(\log y) .
$$

Hence, we obtain that

$$
\begin{aligned}
\int_{x_{n}+c_{1}}^{x_{n}+c_{1}+c} \phi(u) d u & =\int_{x_{n}+c_{1}}^{x_{n}+c_{1}+c} u^{-1-\alpha} h(\log u) d u \\
& \sim x_{n}^{-1-\alpha} \int_{x_{n}+c_{1}}^{x_{n}+c_{1}+c} h(\log u) d u \sim c x_{n}^{-1-\alpha} h(\log y),
\end{aligned}
$$

so that

$$
\int_{x_{n}}^{x_{n}+c} \phi(u) d u \sim \int_{x_{n}+c_{1}}^{x_{n}+c_{1}+c} \phi(u) d u
$$

Case 2. Suppose that $y=x_{0}$. Let $x_{n}+c_{1} \leq u \leq x_{n}+c_{1}+c$ and put

$$
E_{n}:=\left\{u:\left|b^{-m_{n}} u-x_{0}\right| \leq \epsilon b^{-m_{n}}\right\},
$$

where $\epsilon>0$. For sufficiently large $n$, we have for $u \in E_{n}$

$$
-\log \left|b^{-m_{n}} u-x_{0}\right| \geq-\log \epsilon b^{-m_{n}} \geq \frac{1}{2} m_{n} \log b
$$


Set $\lambda_{n}:=\left|y_{n}-x_{0}\right| b^{m_{n}}$. It suffices that we consider the case where there exists a limit of $\lambda_{n}$ as $n \rightarrow \infty$, so we may put $\lambda:=\lim _{n \rightarrow \infty} \lambda_{n}$. This limit permits infinity. We divide $\lambda$ in the two cases where $\lambda<\infty$ and $\lambda=\infty$.

Case 2-1. Suppose that $0 \leq \lambda<\infty$. Now, we have

$$
\begin{aligned}
& \int_{x_{n}+c_{1}}^{x_{n}+c_{1}+c} h(\log u) d u \\
= & \int_{\left[x_{n}+c_{1}, x_{n}+c_{1}+c\right] \backslash E_{n}} h(\log u) d u+\int_{\left[x_{n}+c_{1}, x_{n}+c_{1}+c\right] \cap E_{n}} h(\log u) d u .
\end{aligned}
$$

Let $u \in\left[x_{n}+c_{1}, x_{n}+c_{1}+c\right] \backslash E_{n}$. For sufficiently large $n$, we have by (3.1)

$$
\begin{aligned}
\epsilon b^{-m_{n}} & \leq\left|b^{-m_{n}} u-x_{0}\right| \leq\left|b^{-m_{n}} u-y_{n}\right|+\left|y_{n}-x_{0}\right| \\
& \leq b^{-m_{n}}\left(c+c_{1}\right)+b^{-m_{n}} \lambda_{n} \leq b^{-m_{n}}\left(c+c_{1}+\lambda+1\right) .
\end{aligned}
$$

This implies that

$$
-\log \left|b^{-m_{n}} u-x_{0}\right| \sim m_{n} \log b
$$

For sufficiently large $n$, it follows that

$$
\begin{aligned}
\int_{\left[x_{n}+c_{1}, x_{n}+c_{1}+c\right] \backslash E_{n}} h(\log u) d u & =\int_{\left[x_{n}+c_{1}, x_{n}+c_{1}+c\right] \backslash E_{n}} h\left(\log b^{-m_{n}} u\right) d u \\
& =\int_{\left[x_{n}+c_{1}, x_{n}+c_{1}+c\right] \backslash E_{n}} \frac{-1}{\log \left|b^{-m_{n}} u-x_{0}\right|} d u \\
& \sim \int_{\left[x_{n}+c_{1}, x_{n}+c_{1}+c\right] \backslash E_{n}} \frac{1}{m_{n} \log b} d u
\end{aligned}
$$

As we have

$$
c \geq \int_{\left[x_{n}+c_{1}, x_{n}+c_{1}+c\right] \backslash E_{n}} d u \geq \int_{\left[x_{n}+c_{1}, x_{n}+c_{1}+c\right]} d u-\int_{E_{n}} d u \geq c-2 \epsilon,
$$

it follows that

$$
(1-\epsilon) \cdot \frac{c-2 \epsilon}{m_{n} \log b} \leq \int_{\left[x_{n}+c_{1}, x_{n}+c_{1}+c\right] \backslash E_{n}} h(\log u) d u \leq(1+\epsilon) \cdot \frac{c}{m_{n} \log b}
$$

for sufficiently large $n$. Furthermore, we see from (3.3) that

$$
\begin{aligned}
\int_{\left[x_{n}+c_{1}, x_{n}+c_{1}+c\right] \cap E_{n}} h(\log u) d u & =\int_{\left[x_{n}+c_{1}, x_{n}+c_{1}+c\right] \cap E_{n}} \frac{-1}{\log \left|b^{-m_{n}} u-x_{0}\right|} d u \\
& \leq \frac{2}{m_{n} \log b} \int_{E_{n}} d u \leq \frac{4 \epsilon}{m_{n} \log b} .
\end{aligned}
$$

Hence, we obtain that

$$
\begin{aligned}
\int_{x_{n}+c_{1}}^{x_{n}+c_{1}+c} \phi(u) d u & \sim x_{n}^{-1-\alpha} \int_{x_{n}+c_{1}}^{x_{n}+c_{1}+c} h(\log u) d u \\
& \sim x_{n}^{-1-\alpha} \frac{c}{m_{n} \log b},
\end{aligned}
$$


so that (3.2) holds.

Case 2-2. Suppose that $\lambda=\infty$. For $u$ with $x_{n}+c_{1}+\leq u \leq x_{n}+c_{1}+c$, we see from (3.1) that

$$
\left|y_{n}-x_{0}\right|-\left(c+c_{1}\right) b^{-m_{n}} \leq\left|b^{-m_{n}} u-x_{0}\right| \leq\left|y_{n}-x_{0}\right|+\left(c+c_{1}\right) b^{-m_{n}},
$$

that is,

$$
\left(1-\left(c+c_{1}\right) \lambda_{n}^{-1}\right)\left|y_{n}-x_{0}\right| \leq\left|b^{-m_{n}} u-x_{0}\right| \leq\left(1+\left(c+c_{1}\right) \lambda_{n}^{-1}\right)\left|y_{n}-x_{0}\right| .
$$

This implies that

$$
\begin{aligned}
\int_{x_{n}+c_{1}}^{x_{n}+c_{1}+c} \phi(u) d u & \sim x_{n}^{-1-\alpha} \int_{x_{n}+c_{1}}^{x_{n}+c_{1}+c} \frac{-1}{\log \left|b^{-m_{n}} u-x_{0}\right|} d u \\
& \sim x_{n}^{-1-\alpha} \frac{-c}{\log \left|y_{n}-x_{0}\right|}
\end{aligned}
$$

so we get (3.2). The lemma has been proved.

Lemma 3.2. We have

$$
\phi \otimes \phi(x) \sim 2 M \int_{x}^{x+1} \phi(u) d u=2 M^{2} \mu((x, x+1]) .
$$

Proof Let $\left\{y_{n}\right\}$ be a sequence such that $1 \leq y_{n} \leq b$ and $\lim _{n \rightarrow \infty} y_{n}=y$ for some $y \in[1, b]$. We put $x_{n}=b^{m_{n}} y_{n}$, where $m_{n}$ is a positive integer and $\lim _{n \rightarrow \infty} x_{n}=\infty$. Now, we have

$$
\begin{aligned}
\phi \otimes \phi\left(x_{n}\right) & =\int_{1}^{x_{n}-1} \phi\left(x_{n}-u\right) \phi(u) d u \\
& =2 \int_{1}^{2^{-1} x_{n}} \phi\left(x_{n}-u\right) \phi(u) d u \\
& =2\left(\int_{1}^{\left(\log x_{n}\right)^{\beta}}+\int_{\left(\log x_{n}\right)^{\beta}}^{2^{-1} x_{n}}\right) \phi\left(x_{n}-u\right) \phi(u) d u=: 2\left(J_{1}+J_{2}\right) .
\end{aligned}
$$

Here, we took $\beta$ satisfying $\alpha \beta>1$. Put $K:=\sup \{h(\log x): 1 \leq x \leq b\}$. Then, we have

$$
J_{2} \leq K^{2} \int_{\left(\log x_{n}\right)^{\beta}}^{2^{-1} x_{n}} \frac{d u}{u^{1+\alpha}\left(x_{n}-u\right)^{1+\alpha}} \leq K^{2}\left(\frac{2}{x_{n}}\right)^{1+\alpha} \cdot \alpha^{-1}\left(\log x_{n}\right)^{-\alpha \beta} .
$$

We consider the two cases where $y \neq x_{0}$ and $y=x_{0}$.

Case 1. Suppose that $y \neq x_{0}$. If $1 \leq u \leq\left(\log x_{n}\right)^{\beta}$, then

$$
h\left(\log \left(x_{n}-u\right)\right)=h\left(\log \left(y_{n}-b^{-m_{n}} u\right)\right) \sim h(\log y) .
$$


Hence, we obtain that

$$
\begin{aligned}
J_{1} & =\int_{1}^{\left(\log x_{n}\right)^{\beta}}\left(x_{n}-u\right)^{-1-\alpha} u^{-1-\alpha} h\left(\log \left(x_{n}-u\right)\right) h(\log u) d u \\
& \sim x_{n}^{-1-\alpha} \int_{1}^{\left(\log x_{n}\right)^{\beta}} u^{-1-\alpha} h\left(\log \left(x_{n}-u\right)\right) h(\log u) d u \\
& \sim M x_{n}^{-1-\alpha} h(\log y),
\end{aligned}
$$

so that

$$
\phi \otimes \phi\left(x_{n}\right)=2\left(J_{1}+J_{2}\right) \sim 2 J_{1} \sim 2 M x_{n}^{-1-\alpha} h(\log y) .
$$

Case 2. Suppose that $y=x_{0}$. Put $\gamma_{n}:=b^{m_{n}}\left|y_{n}-x_{0}\right|\left(\log x_{n}\right)^{-\beta}$ and

$$
E_{n}^{\prime}:=\left\{u:\left|y_{n}-x_{0}-b^{-m_{n}} u\right| \leq \epsilon b^{-m_{n}}\right\}
$$

where $0<\epsilon<1$. It suffices that we consider the case where there exists a limit of $\gamma_{n}$, so we may put $\gamma:=\lim _{n \rightarrow \infty} \gamma_{n}$. This limit permits infinity. Furthermore, we divide $\gamma$ in the two cases where $\gamma<\infty$ and $\gamma=\infty$.

Case 2-1. Suppose that $0 \leq \gamma<\infty$. Take sufficiently large $n$. Set

$$
\begin{aligned}
J_{11}^{\prime} & :=\int_{\left[1,\left(\log x_{n}\right)^{\beta}\right] \backslash E_{n}^{\prime}} u^{-1-\alpha} h\left(\log \left(x_{n}-u\right)\right) h(\log u) d u, \\
J_{12}^{\prime} & :=\int_{\left[1,\left(\log x_{n}\right)^{\beta}\right] \cap E_{n}^{\prime}} u^{-1-\alpha} h\left(\log \left(x_{n}-u\right)\right) h(\log u) d u .
\end{aligned}
$$

Let $u \in\left[1,\left(\log x_{n}\right)^{\beta}\right] \backslash E_{n}^{\prime}$. We have

$$
\begin{aligned}
\epsilon b^{-m_{n}} \leq\left|y_{n}-x_{0}-b^{-m_{n}} u\right| & \leq\left|y_{n}-x_{0}\right|+b^{-m_{n}} u \\
& \leq(\gamma+2) b^{-m_{n}}\left(\log x_{n}\right)^{\beta} .
\end{aligned}
$$

This implies that

$$
-\log \left|y_{n}-x_{0}-b^{-m_{n}} u\right| \sim m_{n} \log b
$$

It follows that

$$
\begin{aligned}
J_{11}^{\prime} & =\int_{\left[1,\left(\log x_{n}\right)^{\beta}\right] \backslash E_{n}^{\prime}} u^{-1-\alpha} h\left(\log \left(y_{n}-b^{-m_{n}} u\right)\right) h(\log u) d u \\
& =\int_{\left[1,\left(\log x_{n}\right)^{\beta}\right] \backslash E_{n}^{\prime}} u^{-1-\alpha} h(\log u) \frac{-1}{\log \left|y_{n}-x_{0}-b^{-m_{n}} u\right|} d u \\
& \sim \frac{1}{m_{n} \log b} \int_{\left[1,\left(\log x_{n}\right)^{\beta}\right] \backslash E_{n}^{\prime}} u^{-1-\alpha} h(\log u) d u .
\end{aligned}
$$

Here, we see that, for sufficiently large $n$,

$$
M-\epsilon-2 \epsilon K \leq \int_{\left[1,\left(\log x_{n}\right)^{\beta}\right] \backslash E_{n}^{\prime}} u^{-1-\alpha} h(\log u) d u \leq M,
$$


and thereby

$$
(1-\epsilon) \frac{M-\epsilon-2 \epsilon K}{m_{n} \log b} \leq J_{11}^{\prime} \leq(1+\epsilon) \frac{M}{m_{n} \log b} .
$$

Let $u \in E_{n}^{\prime}$. Then, we have

$$
\begin{aligned}
h\left(\log \left(x_{n}-u\right)\right) & =h\left(\log \left(y_{n}-b^{-m_{n}} u\right)\right) \\
& =\frac{-1}{\log \left|y_{n}-x_{0}-b^{-m_{n}} u\right|} \leq \frac{2}{m_{n} \log b} .
\end{aligned}
$$

Hence, we see that

$$
J_{12}^{\prime} \leq \frac{2}{m_{n} \log b} \int_{\left[1,\left(\log x_{n}\right)^{\beta}\right] \cap E_{n}^{\prime}} u^{-\alpha-1} h(\log u) d u \leq \frac{4 K \epsilon}{m_{n} \log b} .
$$

We consequently obtain that

so that

$$
J_{1} \sim x_{n}^{-1-\alpha}\left(J_{11}^{\prime}+J_{12}^{\prime}\right) \sim \frac{M x_{n}^{-1-\alpha}}{m_{n} \log b},
$$

$$
\phi \otimes \phi\left(x_{n}\right)=2\left(J_{1}+J_{2}\right) \sim 2 J_{1} \sim \frac{2 M x_{n}^{-1-\alpha}}{m_{n} \log b} .
$$

Case 2-2. Suppose that $\gamma=\infty$. Note that $\left[1,\left(\log x_{n}\right)^{\beta}\right] \cap E_{n}^{\prime}$ is empty for sufficiently large $n$. Let $1 \leq u \leq\left(\log x_{n}\right)^{\beta}$. Since

$$
\left|y_{n}-x_{0}\right|\left(1-\gamma_{n}^{-1}\right) \leq\left|y_{n}-x_{0}-b^{-m_{n}} u\right| \leq\left|y_{n}-x_{0}\right|\left(1+\gamma_{n}^{-1}\right) \text {, }
$$

we see that

$$
\log \left|y_{n}-x_{0}-b^{-m_{n}} u\right| \sim \log \left|y_{n}-x_{0}\right|
$$

This yields that

$$
\begin{aligned}
J_{1} & \sim x_{n}^{-1-\alpha} \int_{\left[1,\left(\log x_{n}\right)^{\beta}\right]} u^{-1-\alpha} h(\log u) \cdot \frac{-1}{\log \left|y_{n}-x_{0}-b^{-m_{n}} u\right|} d u \\
& \sim \frac{-M}{\log \left|y_{n}-x_{0}\right|} x_{n}^{-1-\alpha} .
\end{aligned}
$$

For sufficiently large $n$, we have

$$
\begin{aligned}
J_{2} \times x_{n}^{1+\alpha}\left(-\log \left|y_{n}-x_{0}\right|\right) & \leq \frac{2^{1+\alpha} K^{2}}{\alpha} \cdot \frac{-\log \left|y_{n}-x_{0}\right|}{\left(\log x_{n}\right)^{\alpha \beta}} \\
& =\frac{2^{1+\alpha} K^{2}}{\alpha} \cdot \frac{-\log \gamma_{n}+m_{n} \log b-\log \left(\log x_{n}\right)^{\beta}}{\left(\log x_{n}\right)^{\alpha \beta}} \\
& \leq \frac{2^{1+\alpha} K^{2}}{\alpha} \cdot \frac{m_{n} \log b}{\left(\log x_{n}\right)^{\alpha \beta}}
\end{aligned}
$$

so that $\lim _{n \rightarrow \infty} J_{2} / J_{1}=0$. We consequently obtain that

$$
\phi \otimes \phi\left(x_{n}\right)=2\left(J_{1}+J_{2}\right) \sim 2 J_{1} \sim 2 x_{n}^{-1-\alpha} \frac{-M}{\log \left|y_{n}-x_{0}\right|} .
$$


Combining the above calculations with the proof of Lemma 3.1, we reach the following: If $y \neq x_{0}$, then

$$
\phi \otimes \phi\left(x_{n}\right) \sim 2 M x_{n}^{-1-\alpha} h(\log y) \sim 2 M \int_{x_{n}}^{x_{n}+1} \phi(u) d u .
$$

Suppose that $y=x_{0}$. Recall $\lambda$ in the proof of Lemma 3.1. If $0 \leq \gamma<\infty$ and $\lambda=\infty$, then we have $-\log \left|y_{n}-x_{0}\right| \sim m_{n} \log b$. Hence,

$$
\begin{aligned}
\phi \otimes \phi\left(x_{n}\right) & \sim 2 M \frac{x_{n}^{-1-\alpha}}{m_{n} \log b} \\
& \sim 2 M \frac{-x_{n}^{-1-\alpha}}{\log \left|y_{n}-x_{0}\right|} \sim 2 M \int_{x_{n}}^{x_{n}+1} \phi(u) d u .
\end{aligned}
$$

If $0 \leq \gamma<\infty$ and $0 \leq \lambda<\infty$, then

$$
\phi \otimes \phi\left(x_{n}\right) \sim 2 M \frac{x_{n}^{-1-\alpha}}{m_{n} \log b} \sim 2 M \int_{x_{n}}^{x_{n}+1} \phi(u) d u .
$$

If $\gamma=\infty$, then $\lambda=\infty$ and

$$
\phi \otimes \phi\left(x_{n}\right) \sim 2 M \frac{-x_{n}^{-1-\alpha}}{\log \left|y_{n}-x_{0}\right|} \sim 2 M \int_{x_{n}}^{x_{n}+1} \phi(u) d u .
$$

The lemma has been proved.

Proof of Theorem 1.1 We have $\mu \in \mathcal{L}_{\text {loc }}$ by Lemma 3.1. It follows from Lemma 3.2 that

$$
\begin{aligned}
\mu * \mu((x, x+1]) & =M^{-2} \int_{x}^{x+1} \phi \otimes \phi(u) d u \\
& \sim 2 \int_{x}^{x+1} \mu((u, u+1]) d u \sim 2 \mu((x, x+1]) .
\end{aligned}
$$

Let $c>0$. Furthermore, we see from $\mu \in \mathcal{L}_{l o c}$ and (ii) of Lemma 2.2 that

$$
\mu * \mu((x, x+c]) \sim c \mu * \mu((x, x+1]) \text { and } \mu((x, x+c]) \sim c \mu((x, x+1]) .
$$

Hence, we get

$$
\mu * \mu((x, x+c]) \sim 2 \mu((x, x+c]),
$$

and thereby $\mu \in \mathcal{S}_{\text {loc }}$. Thus, $\mu((x-1, x]) \in \mathcal{S}_{d}$ by (i) of Proposition 1.1. Since we see that

$$
\phi \otimes \phi(x) \sim 2 M \int_{x}^{x+1} \phi(u) d u=2 M^{2} \mu((x, x+1]),
$$

we have $\mu^{2 *} \in \mathcal{S}_{a c}$ by (ii) of Lemma 2.1. However, we have $\mu \notin \mathcal{U} \mathcal{L}_{\text {loc }}$ because, for $c=b^{-m(n)}$ with $m(n) \in \mathbb{N}$, we see that as $n \rightarrow \infty$

$$
c^{-1} \int_{b^{n} x_{0}}^{b^{n} x_{0}+c} M^{-1} \phi(u) d u \sim \frac{M^{-1} b^{-(\alpha+1) n} x_{0}^{-\alpha-1}}{(m(n)+n) \log b} .
$$


The above relation implies that the convergence of the definition of the class $\mathcal{U} \mathcal{L}_{\text {loc }}$ fails to satisfy uniformity. Since $\mathcal{U S}_{\text {loc }} \subset \mathcal{U} \mathcal{L}_{\text {loc }}$, the theorem has been proved.

Proof of Corollary 1.1 Proofs of assertions (i) and (ii) are clear from Theorem 1.1. We find from the proof of Theorem 1.1 that $\mu \notin \mathcal{U} \mathcal{L}_{l o c}$ but $\mu^{2 *} \in \mathcal{S}_{a c}$. Since $\mathcal{S}_{a c} \subset \mathcal{L}_{a c} \subset \mathcal{U} \mathcal{L}_{l o c}$, assertions (iii) and (iv) are true.

Choose $x_{1}$ and $x_{2}$ satisfying that $1<x_{0}<x_{0}+x_{1}<x_{0}+x_{2}<b$. Let $\left\{n_{k}\right\}_{k=1}^{\infty}$ be an increasing sequence of positive integers satisfying $\sum_{k=1}^{\infty} 1 / \sqrt{n_{k}}=1$. Let $B_{k}:=$ $\left(-b^{n_{k}} x_{2},-b^{n_{k}} x_{1}\right]$ and $D_{k}:=\left(b^{n_{k}} x_{0}, b^{n_{k}} x_{0}+1\right]$ for $k \in \mathbb{N}$. Choose a distribution $\mu_{1}$ satisfying that $\mu_{1}\left(B_{k}\right)=1 / \sqrt{n_{k}}$ for all $k \in \mathbb{N}$ and $\mu_{1}\left(\left(\cup_{k=1}^{\infty} B_{k}\right)^{c}\right)=0$.

Lemma 3.3. We have, for $c \in \mathbb{R}$,

$$
\lim _{k \rightarrow \infty} \frac{\mu * \mu_{1}\left(D_{k}+c\right)}{\mu\left(D_{k}\right)}=\infty
$$

Proof We have, uniformly in $v \in\left[x_{1}, x_{2}\right]$,

$$
\mu\left(\left(b^{n}\left(x_{0}+v\right), b^{n}\left(x_{0}+v\right)+1\right]\right) \sim M^{-1} b^{-(\alpha+1) n}\left(x_{0}+v\right)^{-\alpha-1} h\left(\log \left(x_{0}+v\right)\right)
$$

and

$$
\mu\left(\left(b^{n} x_{0}, b^{n} x_{0}+1\right]\right) \sim M^{-1} \frac{b^{-(\alpha+1) n} x_{0}^{-\alpha-1}}{n \log b} .
$$

Thus, there exists $c_{1}>0$ such that $c_{1}$ does not depend on $v \in\left[x_{1}, x_{2}\right]$ and that

$$
\liminf _{n \rightarrow \infty} \frac{\mu\left(\left(b^{n}\left(x_{0}+v\right), b^{n}\left(x_{0}+v\right)+1\right]\right)}{n \mu\left(\left(b^{n} x_{0}, b^{n} x_{0}+1\right]\right)} \geq c_{1} .
$$

Hence, we obtain from Lemma 3.1 that

$$
\begin{aligned}
\liminf _{k \rightarrow \infty} \frac{\mu * \mu_{1}\left(D_{k}+c\right)}{\mu\left(D_{k}\right)} & \geq \liminf _{k \rightarrow \infty} \int_{B_{k}} \frac{\mu\left(D_{k}-u+c\right)}{\mu\left(D_{k}\right)} \mu_{1}(d u) \\
& =\liminf _{k \rightarrow \infty} \int_{B_{k}} \frac{\mu\left(D_{k}-u\right)}{\mu\left(D_{k}\right)} \mu_{1}(d u) \\
& \geq c_{1} \liminf _{k \rightarrow \infty} \frac{n_{k}}{\sqrt{n_{k}}}=\infty .
\end{aligned}
$$

Thus, we have proved the lemma.

Proof of Theorem 1.2 Define distributions $\rho_{1}$ and $\rho_{2}$ as

$$
\rho_{1}(d x):=2^{-1} \delta_{0}(d x)+2^{-1} \mu(d x), \quad \rho_{2}(d x):=2^{-1} \mu_{1}(d x)+2^{-1} \mu(d x) .
$$

Thus, $\rho_{1} \in \mathcal{S}_{l o c}$ by Theorem 1.1 and (iii) of Lemma 2.2. Let $\rho(d x):=f(x) d x$, where $f(x)$ is continuous with compact support in $[0,1]$. Define distributions $p_{1}(x) d x$ and $p_{2}(x) d x$ as

$$
p_{1}(x) d x:=\rho * \rho_{1}(d x)=2^{-1} f(x) d x+2^{-1} \rho * \mu(d x)
$$

and

$$
p_{2}(x) d x:=\rho * \rho_{2}(d x)=2^{-1} \rho * \mu_{1}(d x)+2^{-1} \rho * \mu(d x) .
$$


Then, we find that $p_{1}(x)=p_{2}(x)$ for all sufficiently large $x>0$ and $p_{1}(x) \in \mathcal{S}_{d}$ by (ii) of Proposition 1.1. We establish from Lemma 3.3 and Fatou's lemma that

$$
\begin{aligned}
& \liminf _{k \rightarrow \infty} \frac{\int_{D_{k}} p_{2} \otimes p_{2}(x) d x}{\int_{D_{k}} p_{2}(x) d x} \\
& \geq \liminf _{k \rightarrow \infty} \frac{\int_{0}^{2} \mu * \mu_{1}\left(D_{k}-u\right) f^{2 \otimes}(u) d u}{\int_{0}^{1} \mu\left(D_{k}-u\right) f(u) d u} \\
& \geq \int_{0}^{2} \liminf _{k \rightarrow \infty} \frac{\mu * \mu_{1}\left(D_{k}-u\right)}{\mu\left(D_{k}\right)} f^{2 \otimes}(u) d u=\infty .
\end{aligned}
$$

Thus, we conclude that $p_{2}(x) \notin \mathcal{S}_{d}$.

\section{A REMARK ON THE ClOSURE UndeR CONVOLUTION ROOTS}

The tail of a measure $\xi$ on $\mathbb{R}$ is denoted by $\bar{\xi}(x)$, that is, $\bar{\xi}(x):=\xi((x, \infty))$ for $x \in \mathbb{R}$. Let $\gamma \in \mathbb{R}$. The $\gamma$-exponential moment of $\xi$ is denoted by $\widehat{\xi}(\gamma)$, namely, $\widehat{\xi}(\gamma):=\int_{-\infty}^{\infty} e^{\gamma x} \xi(d x)$.

Definition 4.1. $\quad$ Let $\gamma \geq 0$.

(i) A distribution $\rho$ on $\mathbb{R}$ is said to belong to the class $\mathcal{L}(\gamma)$ if $\bar{\rho}(x)>0$ for every $x \in \mathbb{R}$ and if

$$
\bar{\rho}(x+a) \sim e^{-\gamma a} \bar{\rho}(x) \quad \text { for every } \quad a \in \mathbb{R} .
$$

(ii) A distribution $\rho$ on $\mathbb{R}$ belongs to the class $\mathcal{S}(\gamma)$ if $\rho \in \mathcal{L}(\gamma)$ with $\widehat{\rho}(\gamma)<\infty$ and if

$$
\overline{\rho * \rho}(x) \sim 2 \widehat{\rho}(\gamma) \bar{\rho}(x) .
$$

(iii) Let $\gamma_{1} \in \mathbb{R}$. A distribution $\rho$ on $\mathbb{R}$ belongs to the class $\mathcal{M}\left(\gamma_{1}\right)$ if $\widehat{\rho}\left(\gamma_{1}\right)<\infty$.

The convolution closure problem on the class $\mathcal{S}(\gamma)$ with $\gamma \geq 0$ is negatively solved by Leslie [9] for $\gamma=0$ and by Klüppelberg and Villasenor [8] for $\gamma>0$. The same problem on the class $\mathcal{S}_{d}$ is also negatively solved by Klüppelberg and Villasenor [8]. On the other hand, the fact that the class $\mathcal{S}(0)$ of subexponential distributions is closed under convolution roots is proved by Embrechts et al. [5] in the one-sided case and by Watanabe [13] in the two-sided case. Embrechts and Goldie conjecture that $\mathcal{L}(\gamma)$ with $\gamma \geq 0$ and $\mathcal{S}(\gamma)$ with $\gamma>0$ are closed under convolution roots in [3, 4], respectively. They also prove in [4] that if $\mathcal{L}(\gamma) \cap \mathcal{P}_{+}$with $\gamma>0$ is closed under convolution roots, then $\mathcal{S}(\gamma) \cap \mathcal{P}_{+}$with $\gamma>0$ is closed under convolution roots. However, Shimura and Watanabe [12] prove that the class $\mathcal{L}(\gamma)$ with $\gamma \geq 0$ is not closed under convolution roots, and we find that Xu et al. [16] show the same conclusion in the case $\gamma=0$. Pakes [10] and Watanabe [13] show that $\mathcal{S}(\gamma)$ with $\gamma>0$ is closed under convolution roots in the class of infinitely divisible distributions on $\mathbb{R}$. It is still open whether the class $\mathcal{S}(\gamma)$ with $\gamma>0$ is closed under convolution roots. Shimura and Watanabe [11] show that the class $\mathcal{O S}$ is not closed under convolution roots. Watanabe and 
Yamamuro [15] pointed out that $\mathcal{O S}$ is closed under convolution roots in the class of infinitely divisible distributions.

Let $\gamma \in \mathbb{R}$. For $\mu \in \mathcal{M}(\gamma)$, we define the exponential tilt $\mu_{\langle\gamma\rangle}$ of $\mu$ as

$$
\mu_{\langle\gamma\rangle}(d x):=\frac{1}{\widehat{\mu}(\gamma)} e^{\gamma x} \mu(d x)
$$

Exponential tilts preserve convolutions, that is, $(\mu * \rho)_{\langle\gamma\rangle}=\mu\langle\gamma\rangle * \rho_{\langle\gamma\rangle}$ for distributions $\mu, \rho \in \mathcal{M}(\gamma)$. Let $\mathcal{C}$ be a distribution class. For a class $\mathcal{C} \subset \mathcal{M}(\gamma)$, we define the class $\mathfrak{E}_{\gamma}(\mathcal{C})$ by

$$
\mathfrak{E}_{\gamma}(\mathcal{C}):=\left\{\mu_{\langle\gamma\rangle}: \mu \in \mathcal{C}\right\} .
$$

It is obvious that $\mathfrak{E}_{\gamma}(\mathcal{M}(\gamma))=\mathcal{M}(-\gamma)$ and that $\left(\mu_{\langle\gamma\rangle}\right)_{\langle-\gamma\rangle}=\mu$ for $\mu \in \mathcal{M}(\gamma)$. The class $\mathfrak{E}_{\gamma}(\mathcal{S}(\gamma))$ is determined by Watanabe and Yamamuro as follows. Analogous result is found in Theorem 2.1 of Klüppelberg [7].

Lemma 4.1. (Theorem 2.1 of [14]) Let $\gamma>0$.

(i) We have $\mathfrak{E}_{\gamma}(\mathcal{L}(\gamma) \cap \mathcal{M}(\gamma))=\mathcal{L}_{\text {loc }} \cap \mathcal{M}(-\gamma)$ and hence $\mathfrak{E}_{\gamma}\left(\mathcal{L}(\gamma) \cap \mathcal{M}(\gamma) \cap \mathcal{P}_{+}\right)=$ $\mathcal{L}_{\text {loc }} \cap \mathcal{P}_{+}$. Moreover, if $\rho \in \mathcal{L}(\gamma) \cap \mathcal{M}(\gamma)$, then we have

$$
\rho_{\langle\gamma\rangle}((x, x+c]) \sim \frac{c \gamma}{\widehat{\rho}(\gamma)} e^{\gamma x} \bar{\rho}(x) \text { for all } c>0 .
$$

(ii) We have $\mathfrak{E}_{\gamma}(\mathcal{S}(\gamma))=\mathcal{S}_{\text {loc }} \cap \mathcal{M}(-\gamma)$ and thereby $\mathfrak{E}_{\gamma}\left(\mathcal{S}(\gamma) \cap \mathcal{P}_{+}\right)=\mathcal{S}_{\text {loc }} \cap \mathcal{P}_{+}$.

Finally, we present a remark on the closure under convolution roots for the three classes $\mathcal{S}(\gamma) \cap \mathcal{P}_{+}, \mathcal{S}_{l o c} \cap \mathcal{P}_{+}$, and $\mathcal{S}_{a c} \cap \mathcal{P}_{+}$.

Proposition 4.1. The following are equivalent:

(1) The class $\mathcal{S}(\gamma) \cap \mathcal{P}_{+}$with $\gamma>0$ is closed under convolution roots.

(2) The class $\mathcal{S}_{\text {loc }} \cap \mathcal{P}_{+}$is closed under convolution roots.

(3) Let $\mu$ be a distribution on $\mathbb{R}_{+}$and let $p_{c}(x):=c^{-1} \mu((x-c, x])$ for $c>0$. Then, $\left\{p_{c}^{n \otimes}(x): c>0\right\} \subset \mathcal{S}_{d}$ for some $n \in \mathbb{N}$ implies $\left\{p_{c}(x): c>0\right\} \subset \mathcal{S}_{d}$.

Proof Proof of the equivalence between (1) and (2) is due to Lemma 4.1. Let $n \geq 2$. Suppose that (2) holds and, for some $n, p_{c}^{n \otimes}(x) \in \mathcal{S}_{d}$ for every $c>0$. Let $f_{c}(x)=c^{-1} 1_{[0, c)}(x)$. We have $p_{c}^{n \otimes}(x) d x=\left(\left(f_{c}(x) d x\right) * \mu\right)^{n *} \in \mathcal{S}_{l o c}$. We see from assertion (2) that $\left(f_{c}(x) d x\right) * \mu \in \mathcal{S}_{l o c}$ and hence, by (iii) of Proposition 1.1, we have $\mu \in \mathcal{S}_{\text {loc }}$, that is, $p_{c}(x) \in \mathcal{S}_{d}$ for every $c>0$ by (i) of Proposition 1.1. Conversely, suppose that (3) holds and $\mu^{n *} \in \mathcal{S}_{l o c}$. Note that $f_{c}^{n \otimes}(x)$ is continuous with compact support in $\mathbb{R}_{+}$. Thus, we see from (ii) of Proposition 1.1 that $p_{c}^{n \otimes}(x)=$ $\int_{0-}^{x+} f_{c}^{n \otimes}(x-u) \mu^{n *}(d u) \in \mathcal{S}_{d}$ for every $c>0$. We obtain from assertion (3) that $p_{c}(x) \in \mathcal{S}_{d}$ for every $c>0$, that is, $\mu \in \mathcal{S}_{\text {loc }}$ by (i) of Proposition 1.1. 


\section{REFERENCES}

[1] Asmussen, S., Foss, S., Korshunov, D. : Asymptotics for sums of random variables with local subexponential behaviour. J. Theoret. Probab. 16, 489-518 (2003).

[2] Chover, J.; Ney, P.; Wainger, S. : Functions of probability measures. J. Analyse Math. 26, 255-302 (1973).

[3] Embrechts, P., Goldie, C. M. : On closure and factorization properties of subexponential and related distributions. J. Austral. Math. Soc. Ser. A 29, 243-256 (1980)

[4] Embrechts, P., Goldie, C. M. : On convolution tails. Stochastic Process. Appl. 13, 263-278 (1982)

[5] Embrechts, P., Goldie, C. M., Veraverbeke, N. : Subexponentiality and infinite divisibility. Z. Wahrsch. Verw. Gebiete 49, 335-347 (1979)

[6] Foss, S., Korshunov, D., Zachary, S. : An introduction to heavy-tailed and subexponential distributions. Second edition. Springer Series in Operations Research and Financial Engineering. Springer, New York, 2013.

[7] Klüppelberg, C. : Subexponential distributions and characterizations of related classes. Probab. Theory Related Fields 82, 259-269 (1989)

[8] Klüppelberg, C., Villasenor, J. A. : The full solution of the convolution closure problem for convolution-equivalent distributions. J. Math. Anal. Appl. 160, 79-92 (1991)

[9] Leslie, J. R. : On the nonclosure under convolution of the subexponential family. J. Appl. Probab. 26, 58-66 (1989)

[10] Pakes, A. G. : Convolution equivalence and infinite divisibility. J. Appl. Probab. 41, 407-424 (2004)

[11] Shimura, T., Watanabe, T. : Infinite divisibility and generalized subexponentiality. Bernoulli 11, 445-469 (2005)

[12] Shimura, T., Watanabe, T. : On the convolution roots in the convolution-equivalent class. The Institute of Statistical Mathematics Cooperative Research Report 175 pp1-15 2005.

[13] Watanabe, T. : Convolution equivalence and distributions of random sums. Probab. Theory Related Fields 142, 367-397 (2008).

[14] Watanabe, T. Yamamuro, K. : Local subexponentiality and self-decomposability. J. Theoret. Probab. 23, 1039-1067 (2010).

[15] Watanabe, T. Yamamuro, K. : Ratio of the tail of an infinitely divisible distribution on the line to that of its Lévy measure. Electron. J. Probab. 15, 44-74 (2010).

[16] Xu, H, Foss, S., Wang, Y. : Convolution and convolution-root properties of long-tailed distributions. Extremes 18, 605-628 (2015). 